\title{
Mechanisms underlying higher order interactions: from quantitative definitions to ecological processes
}

\author{
Andrew R. Kleinhesselink ${ }^{1}$, Nathan J.B. Kraft ${ }^{1}$, Jonathan M. Levine ${ }^{2}$, \\ ${ }^{1}$ Department of Ecology and Evolutionary Biology, University of California, Los Angeles \\ 621 Charles E. Young Drive South, Los Angeles, USA \\ ${ }^{2}$ Department of Ecology and Evolutionary Biology, Princeton University
}

Running Head: Higher Order Interactions

Key words: resource competition, interaction modification, phenology, annual plants

Submission Type: Article

Main Text Word Count: 6128 (including abstract)

Total Word Count: 8359 (including appendix and literature cited)

Total Pages: 44 (including appendix)

Parts: Main text, Supporting Information Figures and Tables, Appendix A, Computer

Code (zip file) 


\section{Abstract}

2 When species simultaneously compete with two or more species of competitor,

3 higher order interactions (HOIs) can lead to emergent properties not present when species

4 interact in isolated pairs. In order to extend ecological theory to multi-competitor

5 communities, ecologists must develop a practical and general definition for HOIs that can

6 be applied to a wide range of competition models. In this paper we propose a definition

7 for HOIs and outline a set of criteria for testing whether a model has or does not have

8 HOIs. These criteria are valuable for empirical ecologists in need of clarity when

9 discussing HOIs in empirical data. We also provide thorough discussion of how our

10 definition compares with previous definitions of HOIs and interaction modification in the

11 literature. In the second part of the paper we demonstrate the steps required for a rigorous

12 test of HOIs in empirical data. To do this we simulate resource competition between three

13 annual plant species which differ in phenology. We then fit phenomenological

14 competition models to the outcome of simulated competition and use these to test for the

15 presence of HOIs. In our simulations, we find the strength of HOIs varies with

16 phenology: species that grow later experience stronger HOIs than earlier growing species.

17 Our simulation shows how HOIs could emerge in ecosystems where resource availability

18 and individual size change rapidly throughout the course of the growing season and

19 where there are differences in the timing of resource acquisition between competitors. 


\section{Introduction}

22

23

24

25

Almost all species interact with a diversity of predators, pathogens and competitors. Despite this, most classical models in community ecology assume that the per capita effects of each species on each other do not dependent on the densities of any other species in the community. This simplifying assumption means that we can predict the dynamics of multispecies communities from a model that only includes the interaction between each pairs of species (Chesson 2000, Levine et al. 2017).

Higher order interactions (HOIs) between species invalidate the core assumption of independent per capita interactions and thus HOIs could have profound consequences for modeling community dynamics and species coexistence (Neill 1974, Mayfield and Stouffer 2017, Levine et al. 2017, Grilli et al. 2017). If HOIs are strong, even a perfect understanding of the interaction between each and every pair of species in isolation would not be sufficient to describe what happens when all the species are simultaneously interacting (Neill 1974, Billick and Case 1994, Levine et al. 2017). A specific example of the potential for HOIs to impact our understanding of community dynamics is in the application of the mutual invasibility criterion for determining the stability of coexistence (Levine et al. 2017). In theory, HOIs can allow three competitor species to coexist even where some pairs of competitors cannot coexist (Grilli et al. 2017).

Despite the theoretical importance of HOIs, measuring HOIs in nature has been impeded by shifting definitions of what does and does not count as an HOIs (Pomerantz 1981, Adler and Morris 1994, Billick and Case 1994, Letten and Stouffer 2019). Moreover, previous definitions of HOIs were developed with a small range of classical 
43 competition models in mind. Since that time, new statistical modeling software now

44 allows ecologists to fit a much wider range of interaction models (Mayfield and Stouffer

45 2017). This increase in model flexibility requires deriving a more general definition for

46 HOIs that can be applied to any density dependent model of population dynamics.

47 In addition, to the basic issue of producing a shared definition for HOIs,

48 ecologists lack a mechanistic understanding of how HOIs could emerge in nature (Levine

49 et al. 2017, Letten and Stouffer 2019). Such an understanding is necessary for predicting

50 the sets of competitors and ecosystems where strong HOIs are likely. One promising way

51 to address these outstanding issues is to simulate virtual competition experiments based

52 on mechanistic models in which the processes that cause competition are fully known,

53 and then evaluate for which species, and under which conditions HOIs emerge (Letten

54 and Stouffer 2018).

55 We provide a general definition for HOIs based on interaction modification that

56 distinguishes HOIs from related phenomena such as non-linear density dependence and

57 indirect effects. In the second part of the paper, we use a simulation experiment to

58 illustrate how our definition can be applied to properly identify interaction modification

59 even against a backdrop of nonlinear density dependence. We then use the results of the

60 simulation to shed light on possible mechanisms that could generate HOIs in nature.

Higher order interactions result from interaction modification

For the purpose of defining HOIs we focus on modeling a focal species'

performance (usually per capita population growth rate) as a function of the population

density of multiple species of competitor. This can be expressed generally as, 


$$
\frac{1}{n_{i}} \frac{d n_{i}}{d t}=F_{i}\left(n_{1}, \ldots n_{m}\right)
$$

65 where $F_{i}$ gives the per capita population growth rate of the focal species $i$, and $n_{j}$ are the

66

67 81 introduce an interaction modification into the LV model by replacing any of the constant

82 terms $\alpha_{i j}$ with a function of the density of another competitor (Billick and Case 1994).

where, $r_{i}$ is the intrinsic rate of growth of the focal species $i$ and $\alpha_{i j}$ is the per capita effect of competitor $j$ on the growth rate of the focal species. This model is pairwise because each interaction is specified by the pair of species involved, the focal species $i$ and the competitor $j$. The defining property of any pairwise model, such as the LV model, is that the per capita effect of each species of competitor is independent of the densities of any other species of competitor (Figure 1A).

By contrast, interaction modification disrupts pairwise competition and leads to HOIs. Interaction modification occurs when the effect of one competitor species is modified by the density of another competitor species (Adler and Morris 1994). We can terms $\alpha_{i j}$ with a function of the density of another competitor (Billick and Case 1994). 
83 For instance, in the following LV model, the focal species performance is dependent on

84 two competitor species,

$$
\frac{1}{n_{i}} \frac{d n_{i}}{d t}=F_{i}\left(n_{1}, n_{2}\right)=r_{i}\left(1-\alpha_{i 1} n_{1}-\alpha_{i 2} n_{2}\right) .
$$

85 Replacing the term $\alpha_{i 1}$ with the expression $\alpha_{i 1}+\beta_{i(12)} n_{2}$, makes the per capita effect of

86 species one dependent on the density of another competitor, $n_{2}$. More specifically the

87 parameter $\beta_{i(12)}$ measures the strength of this interaction modification (Figure 1B).

88 Substituting this function into the model introduces the product of competitors one and

89 two as a new term,

$$
\frac{1}{n_{i}} \frac{d n_{i}}{d t}=F_{i}^{H O I}\left(n_{1}, n_{2}\right)=r_{i}\left(1-\alpha_{i 1} n_{1}-\alpha_{i 2} n_{2}-\beta_{i(12)} n_{1} n_{2}\right)
$$

90 Interaction modifications such as these imply that competition is functionally different

91 when more than one competitor species is present and that there are emergent properties

92 in the community that cannot be predicted by single species effects. These may suggest

93 specific biological hypotheses: something about the behavior or traits of the competitors

94 are functionally disctinct when they are together as compared to when they are separate.

95 Importantly, an interaction modification cannot be attributed to any one competitor-

96 rather it is an emergent property of the multi-species system, what we call an HOI (Figure

97 1B).

98 An improved general definition of HOIs

99 While the section above captures the essential connection between interaction

100 modifications and HOIs, ecologists do not have a shared definition for HOIs that captures 
101 this idea and which can be applied to any density dependent model of competition

102 (Hairston et al. 1968, Pomerantz 1981, Billick and Case 1994, Grilli et al. 2017, Letten

103 and Stouffer 2019). Here we provide a formal mathematical definition for HOIs rooted in

104 their important implications for ecological theory and which can be applied to any

105 interaction model of any functional form. We first present this more formal definition but

106 follow up with a simple empirical heuristic which can be used to evaluate a model for

107 HOIs.

108 Let $F_{i}\left(n_{1}, \ldots n_{m}\right)$ be a generic model describing the density dependent effects of

$109 m$ competitor species on the per capita growth of species $i$, where $m>1$. Let $\Theta$ be the set

110 of all parameters in the model, $\Theta=\left\{\theta \mid F_{i}\left(n_{1} \ldots n_{m} \mid \theta\right)\right\}$. Here, the term parameter refers

111 to constants in a model that are not themselves dependent variables (Bard 1974). Let

$112 f_{i j}\left(n_{j}\right)$ be a model describing the response of the focal species to competition from a

113 single competitor species, $j$, where $j$ is one of the competitor species included in

$114 F_{i}\left(n_{1}, \ldots n_{m}\right)$. For any model $F_{i}$, we find $f_{i j}\left(n_{j}\right)$ by setting the densities of all

115 competitors except $j$ to zero and simplifying the model. Next, let $\Psi_{j}$ be the set of

116 parameters in $f_{i j}\left(n_{j}\right), \Psi_{\mathrm{j}}=\left\{\psi \mid f_{i j}\left(n_{j} \mid \psi\right)\right\}$. For most realistic competition models the

117 parameters in $\Psi_{\mathrm{j}}$ will be a subset of those in $\Theta$, i.e. $\Psi_{\mathrm{j}} \subseteq \Theta$. Next, let $\Phi$ be the set of all

118 parameters found across all $m$ sets $\Psi_{j}: \Phi=\Psi_{1} \cup \Psi_{2} \cup \ldots \Psi_{m}=\bigcup_{j=1}^{m} \Psi_{j}$. A model is

119 pairwise if all parameters in $\Theta$ are found in the set $\Phi$, i.e. $\Theta=\Phi$. Models with HOIs are

120 defined by having parameters in $F_{i}$ that are not found in the $m$ single-competitor

121 functions, or more precisely, when $\Theta$ is a proper superset of $\Phi, \Theta \supset \Phi$. Finally, let B 
122 be the set of parameters in $\Theta$ but not in $\Phi, \mathrm{B}=\Theta-\Phi$. The parameters in $\mathrm{B}$ are those that

123 define the HOI in the model.

124 As a concrete illustration of our definition, consider the two competitor LV model

125 defined in equation (3): for the full model $\Theta=\left\{\alpha_{i 1}, \alpha_{i 2}, r_{i}\right\}$ and $\Phi=\bigcup_{j=1}^{2} \Psi_{j}=$

$126\left\{\alpha_{i 1}, \alpha_{i 2}, r_{i}\right\}$, thus $\Theta=\Phi$ and the model is pairwise. By contrast, for the HOI model $F_{i}^{H O I}$

127 defined in equation (4), $\Theta=\left\{\alpha_{i 1}, \alpha_{i 2}, \beta_{i(12)}, r_{i}\right\}$ and $\Phi=\bigcup_{j=1}^{2} \Psi_{\mathrm{j}}=\left\{\alpha_{i 1}, \alpha_{i 2}, r_{i}\right\}$, thus

$128 \Theta \supset \Phi$ and the model contains HOIs. Moreover, $\mathrm{B}=\Theta-\Phi=\left\{\beta_{i(12)}\right\}$, thus the

129 parameter $\beta_{i(12)}$ is specifically the one that captures the HOI.

130 This abstract representation belies a simple empirical heuristic for determining

131 whether a model has HOIs: in order to parameterize a model with HOIs, the response of

132 the focal species must be measured against density gradients of each competitor

133 separately, as well as against varying combinations of competitors grown together

134 (Figure 2). This is a natural consequence of the above definition. In essence, a model with

135 HOIs includes additional parameters that an empiricist cannot measure when the

136 response of a focal individual is measured against a single competitor species

137 (Pomerantz 1981). Note, however, there is no way to determine whether there are HOIs

138 among $m$ competitors by examining all $m$ pairwise models $f_{i j}$, rather the form for the

139 multi-competitor model $F_{i}$ must be chosen first in order to apply any HOI definition

140 (Adler and Morris 1994).

141 We refer to the type of HOIs captured by our definition above as hard HOIs and

142 contrast them with the wider phenomenon of non-linear density dependence which

143 produces what we term soft HOIs. A general test for soft HOIs is to take the partial 
144 derivative of the competition function, $F_{i}$ in equation (1), with respect to the density of a

145 single competitor species, $\frac{\partial F_{i}}{\partial n_{j}}$. This partial derivative defines the focal species' sensitivity

146 to a single competitor. If this partial derivative is a function of more than one

147 competitors' density, then there are soft HOIs. In general, all models with hard HOIs will

148 be non-linear and have soft HOIs, but not all non-linear models will have hard HOIs.

149 This is similar to definitions used in earlier discussions of HOIs based on LV forms of

150 competition (Case and Bender 1981), and closely follows the verbal argument that HOIs

151 emerge when the effect of one competitor on another depends on any other competitors.

152 The problem is that any model in which growth is a nonlinear function of

153 interspecific density will involve soft HOIs, and thus this definition does not distinguish

154 interaction modification or HOIs from non-linear density dependence (Pomerantz 1981,

155 Adler and Morris 1994). As an example consider the multi-competitor Hassel model

156 (Hassell and Comins 1976),

$$
\frac{n_{i}(t+1)}{n_{i}(t)}=F\left(n_{1}, \ldots n_{m}\right)=\lambda_{i}\left(1+\sum_{i} \alpha_{i j} n_{j}\right)^{-\tau_{i}}
$$

157 where $\lambda_{i},>0$ is the maximum per capita seed production, $\alpha_{i j}$ is the per capita effect of

158 species $j$ on species $i$ and $\tau_{i}>0$ allows each focal species to respond differently to the

159 sum of competitor effects. This function has the partial derivative $\frac{\partial F_{i}}{\partial n_{j}}=-\alpha_{i j} \lambda_{i}(1+$

$\left.160 \sum_{i} \alpha_{i j} n_{j}\right)^{-\tau_{i}-1}$. Thus, the effect of competitor $j$ on the focal species $i$ is a function of the

161 density of all other competitor species. However, as in the LV model, there are no hard

162 HOIs in this model by our definition because all of the parameters in the multi-competitor 
163 model are also found in the $m$ separate single competitor functions, i.e. $\Theta=\Phi=$

$164\left\{\alpha_{i 1}, \ldots \alpha_{i m}, \lambda_{i}, \tau_{i}\right\}$

165 Why distinguish hard HOIs and non-linear density dependence (soft HOIs)?

166 Hard HOIs and soft HOIs have different interpretations and these differences are

167 important to recognize if we are to advance our understanding of competition in

168 multispecies communities. The question of whether population growth rate declines with

169 competitor density, and whether this decline is linear or non-linear is a longstanding issue

170 in ecology (Hassell and Comins 1976). It would be confusing at best to define HOIs as

171 any non-linear decrease in performance with density - essentially renaming the issue of

172 non-linear density dependence.

173 More importantly, hard HOIs and non-linear density dependence are ecologically

174 distinct as well. Hard HOIs indicate a qualitative change in the way competitors affect a

175 focal species when other competitor species are present. Non-linear density dependence,

176 soft HOIs, does not have the same interpretation. For instance, the net outcome of

177 competition over discrete time intervals may be non-linear when the interaction between

178 competitors is linear in continuous time - the discrete time Hassel model, which is non-

179 linear, is derived from a LV competition model, which is linear in continuous time

180 (Hassell and Comins 1976, O’Dwyer 2018). In the case of the discrete time model, the

181 lifetime competitive effect of each individual declines with competitor density because

182 each individual competitor is smaller and thus has less of an effect on the focal species.

183 Thus, the non-linearity in the model arguably reflects a quantitative not a qualitative 
184 change in the nature of competition when more than one species is present. In models

185 with hard HOIs, the qualitative, or functional change in the nature of competition is

186 defined mathematically by the introduction of additional parameters in $\Theta$ that are not

187 present in $\Phi$ as defined above.

188 Adler and Morris (1994) provide another specific example where it is ecologically

189 meaningful to differentiate between HOIs and non-linear density dependence. They

190 describe a hypothetical scenario in which different species of plants compete for light and

191 each species simply blocks a proportion of the light that passes through its canopy-thus

192 taller species reduce the amount of light received by shorter species. In this way, the

193 qualitative nature of the interaction between a tall species and a shorter one is

194 independent of all other species. Nevertheless, this mechanism of interaction means that

195 the effect of a taller species on a shorter species below it depends non-additively on the

196 density of other competitors with a canopy between the two. Per capita competition is

197 non-additive, but arguably there is no ecologically distinct interaction modification

198 between the different competitors - they simply reduce the fraction of light received

199 regardless of the presence of other species. By contrast, hard HOIs as we define them

200 introduce new parameters, or new functional dependencies, between competitors that

201 only kick in when more than one competitor is present.

202 Our definition also helps resolve the question of whether single species effects

203 can involve HOIs. For instance, recent papers by Letten and Stouffer (2019) and

204 Mayfield and Letten (2017) define HOIs as any higher order terms of competitor density, 205 including single species quadratic terms, $\beta_{i(j j)} N_{j}^{2}$. Our definition, does not count these as 
206 HOIs, and this agrees with the emphasis in the literature that HOIs are a phenomenon that

207 arises between two or more different species of competitor (Hairston et al. 1968,

208 Vandermeer 1969, Neill 1974, Morin et al. 1988). As per our definition, the coefficients

209 for these terms, $\beta_{i(j j)}$, are parameters in a pairwise model, $f_{i j}\left(n_{j}\right)$, and thus are not hard

210 HOIs. Nor can single species higher order terms (not to be confused with higher order

211 interactions) generally be interpreted as examples of intraspecific interaction

212 modification, i.e. the effect of each additional individual being modified by other

213 individuals of the same species (Mayfield and Stouffer 2017). This interpretation only

214 makes sense in the context of a model where density dependence is strictly linear. In non-

215 linear models, such as those fit in Mayfield and Stouffer (2017), higher order terms added

216 to the model cannot be interpreted as individual-level interaction modifications; rather

217 these additional terms simply allow an already non-linear function to more closely

218 approximate the observed relationship between density and performance.

219 Another definition for HOIs that is largely equivalent to ours is provided by Adler

220 and Morris (1994). Like our definition, Adler and Morris distinguished between HOIs

221 and non-linear density dependence, and their definition agrees with ours in most cases.

222 However, there are some cases with three or more competitor species where the Adler

223 and Morris approach would indicate an HOI and our definition would not. We believe

224 our definition is more general, it does not depend on the number of competitor species

225 present and it can be more directly related to the traditional verbal definitions that

226 ecologists have used when discussing HOIs. 
In the remainder of this paper we outline the experimental set-up and statistical

228 analyses required to test for HOIs in empirical data. Because real world data that would

229 allow for rigorous tests of HOIs are limited, we use a mechanistic growth model to

230 simulate a virtual competition experiment among three annual plant species (Figure 3).

231 We then fit species' responses to interspecific competition using phenomenological

232 competition models with and without HOIs and evaluate which species' responses are

233 best fit by competition model with HOIs. By considering when HOIs emerge in this

234 simple simulation we show the steps required to detect HOIs in empirical data and shed

235 light on the processes that could generate HOIs in nature.

\section{Simulating a Higher Order Competition Experiment}

A rigorous demonstration of HOIs requires measuring how focal species'

238 performance changes in response to increasing densities of each competitor species in

239 isolation, as well as to varying densities of combinations of different competitor species.

240 This requires an orthogonal response surface design where each competitor's density is

241 varied independently of each other species.

242 Instead of analyzing real data, we used a mechanistic growth model to simulate a

243 virtual experiment in which individuals of each annual plant species are grown in

244 separate plots with a range of competitor densities (Figure 2). The simulation lasts one

245 growing season (200 days). After the simulation ends, we find the per capita seed output

246 of each focal individual and record this as a measure of performance. We quantified

247 performance in plots with densities of $0,1,2,3,4,9,16,25$ or 36 individuals of each 
248 other competitor species, including intraspecific competition. We also measured

249 performance when the focal species was grown against all possible combinations of two

250 competitor species at the same densities. This design allows us to fit non-linear functions

251 to the interaction between each pair of species and test for any HOIs when more than two

252 competitors are present together.

253 We developed a mechanistic growth and resource competition model intended to

254 simulate the growth of annual plants in a Mediterranean climate (Figure S 1). The

255 simulated individuals germinate in the winter and then grow, flower, and produce seeds

256 by the early summer (Godoy and Levine 2014). In our model, we track a single pool of

257 soil resources, most easily thought of as water or water-soluble nutrients. This pool is not

258 resupplied during the season and is depleted over time. As the resource concentration

259 declines, plant growth slows and eventually stops (Figure 3). We make the assumption

260 that when individual net growth is zero, the plant will convert a fraction of its biomass

261 into seeds that remain dormant until the start of the next growing season (Cohen 1976).

262 Assuming all seeds germinate at the same time, and no seed mortality, we can use the per

263 capita seed production as a direct measure of population growth rate in each competition

264 treatment.

265 Resource dynamics in the model are given by the differential equation,

$$
\frac{d R}{d u}=I-\sum_{i=1}^{m} n_{i} g_{i}\left(b_{i}\right) h(R(u)),
$$

266 where $R(u)$ is the resource availability at time $u$ ( $u$ being day within the growing

267 season), $I$ is the resource supply rate, and the final term is the sum of resource uptake 
268 rates of all $m$ species in the community. Biomass per individual of each species $i$ at time

$269 u$ is given by $b_{i}$ and the number of individuals in the population is given by $n_{i}$. The

270 function $g_{i}\left(b_{i}\right)$ converts per capita biomass into surface area of fine roots. Total resource

271 uptake rate is the product of root surface area and the rate of resource conductance per

272 unit root surface area. The rate of resource conductance into the roots is a function, $h(R)$,

273 of soil resource concentration, which we specify below. We simulate a Mediterranean

274 climate by setting initial resource availability high, $R(u=0) \gg 0$, and setting the

275 resource supply rate, $I$, to zero.

276 Growth of each species is given by a piecewise differential equation,

$$
\frac{d B_{i}}{d u}=\left\{\begin{aligned}
n_{i} g_{i}\left(b_{i}\right) h(R) q-\delta_{i} B_{i}, & n_{i} g_{i}\left(b_{i}\right) h(R) q>\delta_{i} B_{i} \\
0, & n_{i} g_{i}\left(b_{i}\right) h(R) q \leq \delta_{i} B_{i}
\end{aligned}\right.
$$

277 where, $q$ is the rate of resource conversion into biomass and $\delta_{i}$ is the rate of biomass loss

278 and respiration. The conditions indicate that when net growth of each species is less than

279 or equal to zero, growth and resource consumption stops (i.e. is set to zero). Biomass per

280 individual plant, $b_{i}$, is converted into root surface area for each individual via the function

$281 g_{i}\left(b_{i}\right)=\left(\frac{p b_{i}}{d_{i}}\right)^{v}$, where $p$ is the proportion of growth allocated to roots, $d_{i}$ is root tissue

282 density in $\mathrm{g} \mathrm{cm}^{-3}$ and $v$ is an exponent that scales root volume to root surface area (see

283 Kooijmans (1986) for a conceptually similar approach to protists). The rate of resource

284 uptake per unit root surface area is dependent on resource concentration following

285 Michaelis-Menton kinetics:

$$
h(R)=\frac{V_{\max } R}{K+R} .
$$


The equations above describe growth in total population biomass, $B_{i}$, over the

287 course of days within a single growing season. In contrast, a population-level

288 phenomenological competition model would track the total population density, $n_{i}$, over

289 annual time steps, $t$. In order to convert population density into biomass, we assume that

290 individuals start the growing season as seeds with a fixed size. Thus, the initial biomass

291 is $B_{i}(0)=\mu n_{i}(t)$, where $\mu$ is mass per seed and $n_{i}(t)$ is the number of seeds in the

292 population in year $t$. The population density in the following year $n_{i}(t+1)$ is the total

293 number of seeds produced by the mature plants at the end of the growing season,

$$
n_{i}(t+1)=\frac{c}{\mu}\left(\max B_{i}\right)
$$

294 where $\max B_{i}$ is the final accumulated biomass of species $i$ and $c$ gives the proportion of 295 total biomass converted to seeds.

296 We simulate the dynamics of three virtual annual plant species that differ in their 297 allocation to roots and in their rates of resource uptake (Table S 1). This difference leads 298 to phenology differences, i.e. some species stop growing earlier than others (Figure 3).

299 Phenology differences emerge because of the assumed trade-off between species rank in 300 terms of root density $d_{i}$ and rank in terms of tissue respiration and loss rate, $\delta_{i}$, (Tjoelker 301 et al. 2005, Birouste et al. 2014) (Table S1). Species with lower root density convert each 302 gram of biomass into more root surface area and this allows them to grow faster early in 303 the season when resource concentrations are high. In contrast, species with denser roots 304 but lower rates of tissue loss and respiration grow more slowly but continue growing later 305 into the season as resource availability declines. Thus, we refer to the three species in our 
306 simulations as 'early', 'mid' and 'late', depending on when they stop growing during the

307 simulation (Figure 3).

308 We chose parameters that produced growth and phenology patterns qualitatively

309 similar to biomass accumulation curves observed in annual plant communities (Godoy

310 and Levine 2014). A table of parameter values for the simulations are provided in the

311 supporting information (Table S 1). We simulated growth and resource dynamics by

312 solving equations (6) and (7) with the package deso 1 ve in the statistical program $\mathrm{R}(\mathrm{R}$

313 Core Team 2015). Code to reproduce analyses is available in a zip file and on github:

314 https://github.com/akleinhesselink/Competitive_HOI/releases/tag/1.0

315 Phenomenological annual plant model

316 In order to investigate whether this simulation produces HOIs between the

317 competitors, we fit non-linear phenomenological competition models to the per capita

318 seed production of each species. After evaluating a number of non-linear models, we

319 found that the Hassel model (Eq. [5]) fit the outcome of simulated pairwise competition

320 well. We specified an HOI version of the Hassel model as follows,

$$
\frac{n_{i}(t+1)}{n_{i}(t)}=\frac{\lambda_{i}}{\left(1+\sum_{j=1}^{3} \alpha_{i j} n_{j}+\sum_{j=1}^{3} \sum_{k=j+1}^{3} \beta_{i(j k)} n_{j} n_{k}\right)^{\tau_{i}}},
$$

321 where all HOI effects of two competitor species on the focal species $i$ are fitted with the

322 coefficients $\beta_{i(j k)}$ (following the notation in Mayfield and Stouffer (2017)). By our

323 definition, $\beta_{i(j k)}$ is a hard HOI when $j \neq k$.

324 Finally, we also considered a pairwise multiplicative version of the Hassel form, 


$$
\frac{n_{i}(t+1)}{n_{i}(t)}=\frac{\lambda_{i}}{\left(\prod_{j=1}^{3}\left(1+\alpha_{i j} n_{j}\right)\right)^{\tau_{i}}}
$$

325 This model does not have HOIs per our definition - all $\alpha_{i j}$ and $\tau_{i}$ parameters can be

326 estimated from the pairwise cases where the focal species $i$ competes with each other

327 species $j$ in isolation. However, when there are two or more competitors the denominator

328 becomes a polynomial with multiplicative terms of competitor density. In the case of only

329 one competitor species, it collapses to the same pairwise Hassel model. Thus, contrasting

330 this model with the HOI model allows us to test whether hard HOIs are required as

331 opposed to a simpler non-linear function without HOIs.

332 We first fit the Hassel model to the pairwise cases and checked the model fit

333 graphically. We then fit the Hassel models (Eq. [5]), the HOI model (Eq. [10]) and the

334 multiplicative pairwise model (Eq. [11]) to the full set of two competitor densities. For

335 each focal species and model, we calculated root mean squared error (RMSE) as a

336 measure of goodness of fit and evaluated the strength and direction of HOIs by

337 examining the HOI coefficients, $\beta_{i(j k)}$. We fit all models with the non-linear least squares

338 modelling function, $\mathrm{n} 7 \mathrm{~s}$, in $\mathrm{R}$. Code to run the simulations, fit the models and produce

339 the figures is given in the online supporting information.

\section{Results}

For all three species we found the Hassel model fit the simulated pairwise data

342 accurately (Figure 4). Next, we compared the three models fit to the full range of

343 competitor densities (Figure 5). For the early season species, the Hassel model with and

344 without the HOI showed more or less equivalent fits to the data with only a slight 
345 decrease in RMSE for the HOI model (Figure 5A\&G). For the mid-season and late-

346 season species, we found that the HOI model fit the data better than the pairwise Hassel

347 model (Figure 5 B-I). The inability of the pairwise Hassel model to fit the per capita seed

348 output of the mid and late-season species can be seen by plotting the observed and per

349 capita seed production against two competitor densities at once (Figure S 2). In all cases,

350 the fitted HOI coefficients, $\beta_{i(j k)}$, were of smaller magnitude than the fitted pairwise

351 effects, $\alpha_{i j}$ (Figure 6). The fitted HOIs were stronger for the mid and late season species

352 than for the early season species (Figure 6). The multiplicative model (Eq. [11]) fit the

353 multi-competitor dynamics poorly when compared to the pairwise model and the HOI

354 model (Figure 5).

\section{Discussion}

\section{Evidence for higher order interactions}

357 Our simulation shows clear evidence for HOIs affecting two of the three virtual

358 species in our simulations (Figure 6). For the mid and late season species, the functional

359 form of per capita competition changed depending on the presence of other interspecific

360 competitors. Specifically, the presence of early or mid-season competitors increased the

361 per capita effects of competition on the late-season species (Figure 6F). Likewise, the

362 presence of the early season species increased the per capita effects of competition on the

363 mid-season species (Figure 6E). For the early season species, no clear HOIs were

364 detected: the pairwise interaction Hassel model fit the data nearly as well as the HOI 
365 model (Figure 5A\&G) and the magnitudes of the HOI coefficients were small (Figure

366 6D).

367 We observe competition in our simulations because of a feedback between

368 individual density and resource concentration. As individuals grow, they draw down

369 resource concentrations (Figure S 1), this decreases the rate of resource acquisition into

370 the roots by equation (8) and slows the growth of other individuals in the community.

371 The magnitudes of pairwise interactions between species is easily understood from this

372 perspective. For instance, the late season species has a weak per capita effect on the early

373 season species because while the early species is active, roughly day 0 to day 30 , the late-

374 season species remains small and has a slow absolute rate of resource uptake (Figure

$3753 \mathrm{~A}$-blue line ). In contrast, the mid-season species has a stronger effect on the early

376 season species because it grows faster during the same period (Figure 3-red line). On

377 the other hand, the early season species has a weak effect on the late season species

378 because the former stops growing before the latter does the majority of its growth (Figure

3793 -black line).

380 The simplicity of the simulation makes it possible to understand how HOIs

381 emerge as well. The HOIs that affect the mid and late season species are in part due to an

382 indirect effect of resource uptake on competitor size and in part due to changes in

383 competitor phenology. For instance, in a scenario with one individual of each species the

384 early season species slows the growth of both the mid and the late-season species, this

385 keeps them smaller later into the season and makes them both more sensitive to

386 competition as the season progresses (Figure 3). This is reflected in the HOI coefficients 
387 that magnify competition for the mid and late-season species (Figure 6E\&F). In contrast,

388 the early season species grows fast and exerts the dominant effect on the resource while it

389 is active, this makes it relatively insensitive to changes in the size of its interspecific

390 competitors (Figure 6D).

391 While the HOIs in this system are similar to competition mediated indirect effects

392 (Levine et al. 2017) there are two important differences between the HOIs we observed

393 and traditional indirect effects. First, indirect effects are not emergent properties of a

394 multi-competitor system, rather they are a predictable result of pairwise per capita

395 competition coefficients (Kleinhesselink and Adler 2015). Second, indirect effects can

396 generally be understood as emerging because of changes in the density of competitors

397 over time, not because of changes in per capita competition. For example, one species

398 may have an indirect effect on its competitor by changing the density of a second

399 competitor over the course of several year. In contrast, the HOIs in our simulation emerge

400 over the course of a single growing season with fixed population densities. Thus, these

401 HOIs indicate ecologically meaningful changes in the per capita effect of one species on

402 another.

403 Our example can be contrasted with a recent simulation of forest dynamics that

404 demonstrated how HOIs could affect species coexistence (Grilli et al. 2017). In that

405 simulation, unlike ours, per capita interactions between species were fixed. What the

406 authors called HOIs in that model, were not due to changes in the per capita effect of

407 competition, but were caused by changes in competitor density over time that were not

408 explicitly tracked by the model. 


\section{The phenomenological nature of HOIs}

HOIs can only be defined and quantified within the context of phenomenological models of competition. Phenomenological models simplify community dynamics by tracking population densities and not the resources for which species compete (Chesson 2000). HOIs emerge in phenomenological models precisely because they leave out mechanistic detail and do not explicitly model resource dynamics (Abrams 1983, O’Dwyer 2018, Letten and Stouffer 2019). Given this, one may be tempted to conclude that HOIs are an artifact of the inadequacy of such models. However, any concept of species interactions (at least competitive interactions) is essentially phenomenological in nature-biomass and nutrients do not flow directly between competing individuals, rather competitors influence each other's growth or survival indirectly through changes in the abundance of shared resources. Thus one could sidestep the problem of HOIs by instead modeling communities mechanistically as biomass and resources (e.g. Dybzinski and Tilman (2007)). However, doing may require re-thinking ecological theory formulated on the concept of species interactions.

Phenomenological competition coefficients can sometimes be derived analytically from mechanistic competition models by making the assumption that resource concentrations are near a fixed equilibrium (Tilman 1977, Meszéna et al. 2006, Kleinhesselink and Adler 2015, Letten et al. 2017). However, in many natural systems, such as such as those involving annual plants, resource concentrations and individual size fluctuate rapidly over the course of a single growing season or generation. This makes deriving competition coefficients directly from the resource dynamics more difficult, 
431 perhaps impossible (O’Dwyer 2018). Thus, even in cases in which we actually know

432 which resources species compete for, fitting a phenomenological model to population

433 dynamics may be the only way to quantitively describe species interactions. Our work

434 clarifies the what it means to fit models with and without HOIs to multi-competitor

435 settings.

436 Are HOIs widespread?

437 In our virtual experiment, HOIs arise because individual size and phenology, the

438 traits that determine each species' impact on and sensitivity to resource availability, are

439 themselves governed by resource availability. More generally, changes in individual size

440 and corresponding changes in resource uptake rate may be a common cause of HOIs in

441 nature. We predict that HOIs will likely be common in systems in which 1) consumers

442 cause large resource fluctuations, 2) the per capita rate of resource uptake changes in

443 response to resource availability, and 3) the strength of this response varies across

444 species. Instead of changes in individual size, another mechanism that could generate

445 HOIs would be density-dependent changes in resource acquisition traits. For example,

446 traits such as height, specific leaf area, and phenology, have been shown to change in

447 response to competition or resource availability (e.g. Aronson et al. 1992, Bennett et al.

448 2016, Conti et al. 2018). If per capita competition coefficients are a function of these

449 traits, then it would not be surprising if changes in these traits led to HOIs. If changes in

450 individual size within a season, or trait plasticity are common, and are also likely to cause

451 HOIs, this begs the question of why there have been so few documented examples of

452 HOIs in natural communities (but see Mayfield and Stouffer 2017). 
One hypothesis is that HOIs are common but usually too weak to detect. A key

454 factor in producing HOIs in our simulation is that each species has a uniquely shaped

455 growth curve and phenology. In additional simulations, we found that as species became

456 more similar in their traits HOIs became weaker (Appendix A). In nature, such large

457 functional differences in the way species take-up resources over time may be rare. At the

458 same time, these simulations suggest that quantifying how functional traits change in

459 response to competitors provides a likely path to further understanding of HOIs.

460 A second factor generating the HOIs in our simulation are the rapid changes in

461 resource availability and average plant size, and consequently, species interactions, over

462 the course of a season (Figure 3). Without these dynamics, species might have relatively

463 constant per capita effects on one another and no HOIs would emerge. For instance,

464 compare our system to an idealized version of resource competition for perennial plants

465 (Dybzinski and Tilman 2007). Due to their large size perennial plants can be assumed to

466 quickly draw resources down to a dynamic equilibrium. By contrast, seasonally forced

467 systems such as annual plant communities in Mediterranean climates may be a good

468 place to look for strong HOIs (Mayfield and Stouffer 2017).

\section{Conclusion}

HOIs have profound implications for how we understand and model multispecies

471 communities. However, before ecologists can embark on measuring HOIs in nature, they

472 must have a shared definition for what HOIs are. We have provided a more general

473 definition of HOIs caused by interaction modifications that will be useful as ecologists 
474 seek empirical evidence for HOIs in nature. By simulating growth and resource

475 competition in a virtual experiment, we outline the steps required to fit pairwise and HOI

476 models to field data. This simulation also sheds light on the environmental conditions and

477 life-history traits that may be more likely to generate HOIs. While we believe that HOIs

478 should be common in nature this does not mean that they will be strong enough to detect

479 statistically. Our work suggests that environments in which resource availability and

480 competitor size change rapidly during a single growing season may be a likely place for

481 detectable HOIs to emerge.

\section{Acknowledgments}

483 We wish to thank Joanna Shih for providing the botanical line drawings. Gaurav

484 Kandlikar, Theo Gibbs, Chris Klausmeier, two anonymous reviewers and members of the

485 Kraft and Levine lab provided valuable comments on earlier drafts of this manuscript. 


\section{References}

487 Abrams, P. A. 1983. Arguments in Favor of Higher Order Interactions. The American $488 \quad$ Naturalist 121:887-891.

489 Adler, F. R., and W. F. Morris. 1994. A General Test for Interaction Modification.

$490 \quad$ Ecology $75: 1552-1559$.

491 Aronson, J., J. Kigel, A. Shmida, and J. Klein. 1992. Adaptive phenology of desert and 492 Mediterranean populations of annual plants grown with and without water stress. 493 Oecologia 89:17-26.

494 Bard, Y. 1974. Nonlinear parameter estimation. Academic Press, New York, NY.

495 Bennett, J. A., K. Riibak, R. Tamme, R. J. Lewis, and M. Pärtel. 2016. The reciprocal 496 relationship between competition and intraspecific trait variation. Journal of $497 \quad$ Ecology 104:1410-1420.

498 Billick, I., and T. J. Case. 1994. Higher Order Interactions in Ecological Communities:

499 What Are They and How Can They be Detected? Ecology 75:1530-1543.

500 Birouste, M., E. Zamora-Ledezma, C. Bossard, I. M. Pérez-Ramos, and C. Roumet. 2014.

501 Measurement of fine root tissue density: a comparison of three methods reveals 502 the potential of root dry matter content. Plant and Soil 374:299-313.

503 Case, T. J., and E. A. Bender. 1981. Testing for Higher Order Interactions. The American $504 \quad$ Naturalist 118:920-929.

505 Chesson, P. 2000. Mechanisms of Maintenance of Species Diversity. Annual Review of $506 \quad$ Ecology and Systematics 31:343-366. 
507 Cohen, D. 1976. The Optimal Timing of Reproduction. The American Naturalist

$508 \quad 110: 801-807$.

509 Conti, L., S. Block, M. Parepa, T. Münkemüller, W. Thuiller, A. T. R. Acosta, M. van

510 Kleunen, S. Dullinger, F. Essl, I. Dullinger, D. Moser, G. Klonner, O. Bossdorf,

511 and M. Carboni. 2018. Functional trait differences and trait plasticity mediate

512 biotic resistance to potential plant invaders. Journal of Ecology 106:1607-1620.

513 Dybzinski, R., and D. Tilman. 2007. Resource Use Patterns Predict Long-Term

514 Outcomes of Plant Competition for Nutrients and Light. The American Naturalist $515 \quad 170: 305-318$.

516 Godoy, O., and J. M. Levine. 2014. Phenology effects on invasion success: insights from 517 coupling field experiments to coexistence theory. Ecology 95:726-736.

518 Grilli, J., G. Barabás, M. J. Michalska-Smith, and S. Allesina. 2017. Higher-order 519 interactions stabilize dynamics in competitive network models. Nature 548:210$520 \quad 213$.

521 Hairston, N. G., J. D. Allan, R. K. Colwell, D. J. Futuyma, J. Howell, M. D. Lubin, J. 522 Mathias, and J. H. Vandermeer. 1968. The Relationship between Species 523 Diversity and Stability: An Experimental Approach with Protozoa and Bacteria. $524 \quad$ Ecology 49:1091-1101.

525 Hassell, M. P., and H. N. Comins. 1976. Discrete time models for two-species 526 competition. Theoretical Population Biology 9:202-221.

527 Kleinhesselink, A. R., and P. B. Adler. 2015. Indirect effects of environmental change in 528 resource competition models. The American Naturalist 186:766-776. 
529 Kooijman, S. A. L. M. 1986. Energy budgets can explain body size relations. Journal of

$530 \quad$ Theoretical Biology 121:269-282.

531 Letten, A. D., P.-J. Ke, and T. Fukami. 2017. Linking modern coexistence theory and 532 contemporary niche theory. Ecological Monographs 87:161-177.

533 Letten, A. D., and D. B. Stouffer. 2019. The mechanistic basis for higher-order 534 interactions and non-additivity in competitive communities. Ecology Letters $535 \quad 22: 423-436$.

536 Levine, J. M., J. Bascompte, P. B. Adler, and S. Allesina. 2017. Beyond pairwise mechanisms of species coexistence in complex communities. Nature 546:56-64.

538 Mayfield, M. M., and D. B. Stouffer. 2017. Higher-order interactions capture unexplained complexity in diverse communities. Nature Ecology \& Evolution

Meszéna, G., M. Gyllenberg, L. Pásztor, and J. A. J. Metz. 2006. Competitive exclusion and limiting similarity: A unified theory. Theoretical Population Biology 69:68Insects and Vertebrates: Interaction Strength and Higher Order Interactions.

547 Neill, W. E. 1974. The Community Matrix and Interdependence of the Competition Coefficients. The American Naturalist 108:399-408.

549 O’Dwyer, J. P. 2018. Whence Lotka-Volterra?: Conservation laws and integrable systems in ecology. Theoretical Ecology. 
551 Pomerantz, M. J. 1981. Do "Higher Order Interactions" in Competition Systems Really

$552 \quad$ Exist? The American Naturalist 117:583-591.

553 R Core Team. 2015. R: A Language and Environment for Statistical Computing. R

$554 \quad$ Foundation for Statistical Computing, Vienna, Austria.

555 Tilman, D. 1977. Resource Competition between Plankton Algae: An Experimental and

$556 \quad$ Theoretical Approach. Ecology 58:338-348.

557 Tjoelker, M. G., J. M. Craine, D. Wedin, P. B. Reich, and D. Tilman. 2005. Linking leaf

558 and root trait syndromes among 39 grassland and savannah species. New

$559 \quad$ Phytologist 167:493-508.

560 Vandermeer, J. H. 1969. The Competitive Structure of Communities: An Experimental

$561 \quad$ Approach with Protozoa. Ecology 50:362-371. 


\section{Figures}

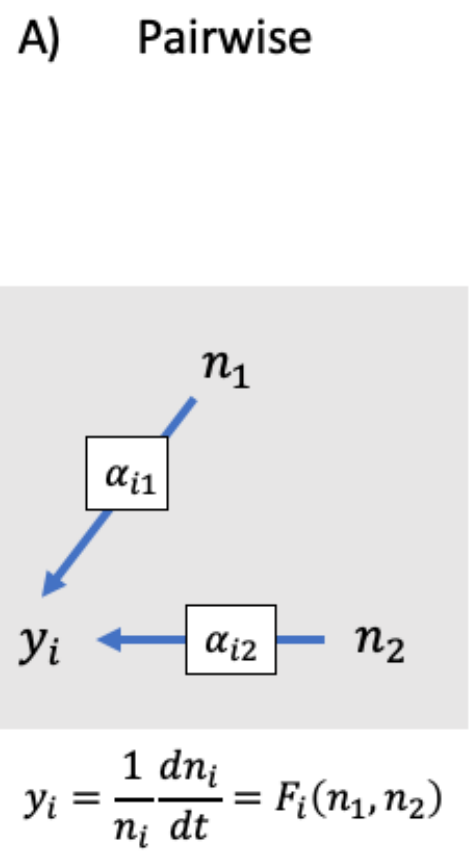

B) Interaction Modification
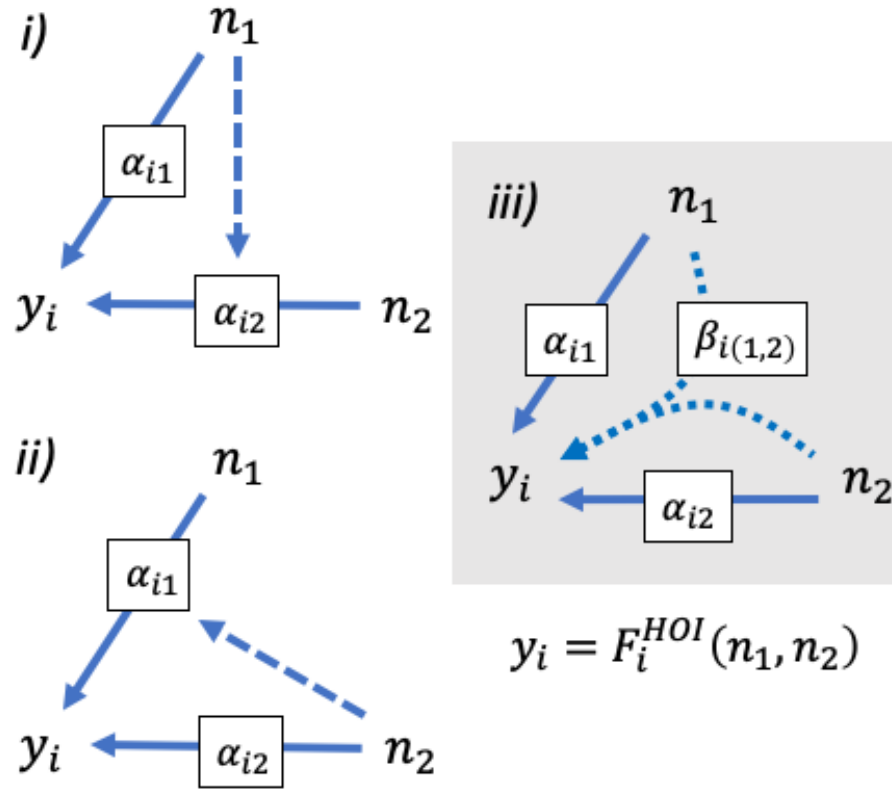

565 Figure 1. How interaction modifications lead to higher order interactions. In A, a

566 pairwise model is shown without interaction modification. The competitive effect of

567 species one and two on the per capita growth of the focal species $i$, are shown as

568 separate blue arrows. These effects may be simple per competition coefficients, $\alpha_{12}$

569 and $\alpha_{13}$, or could be more complicated non-linear functions of density. In B, a

570 model with interaction modification is shown: in $i)$ the dashed arrow shows that the

571 effect of two is modified by the density of one; in ii) the effect of one is modified by

572 the density of two. In reality, one cannot assign either species as the modifier, rather 
573 they modify each other's effects in a way that emerges a single HOI. The HOI in

574 this case is quantified by introducing the new parameter $\beta_{i(1,2)}$, and shown with the 575 curved arrows in iii. 


\section{Performance \\ of focal alone}

COMPETITOR 1$$
\text { 西 }
$$
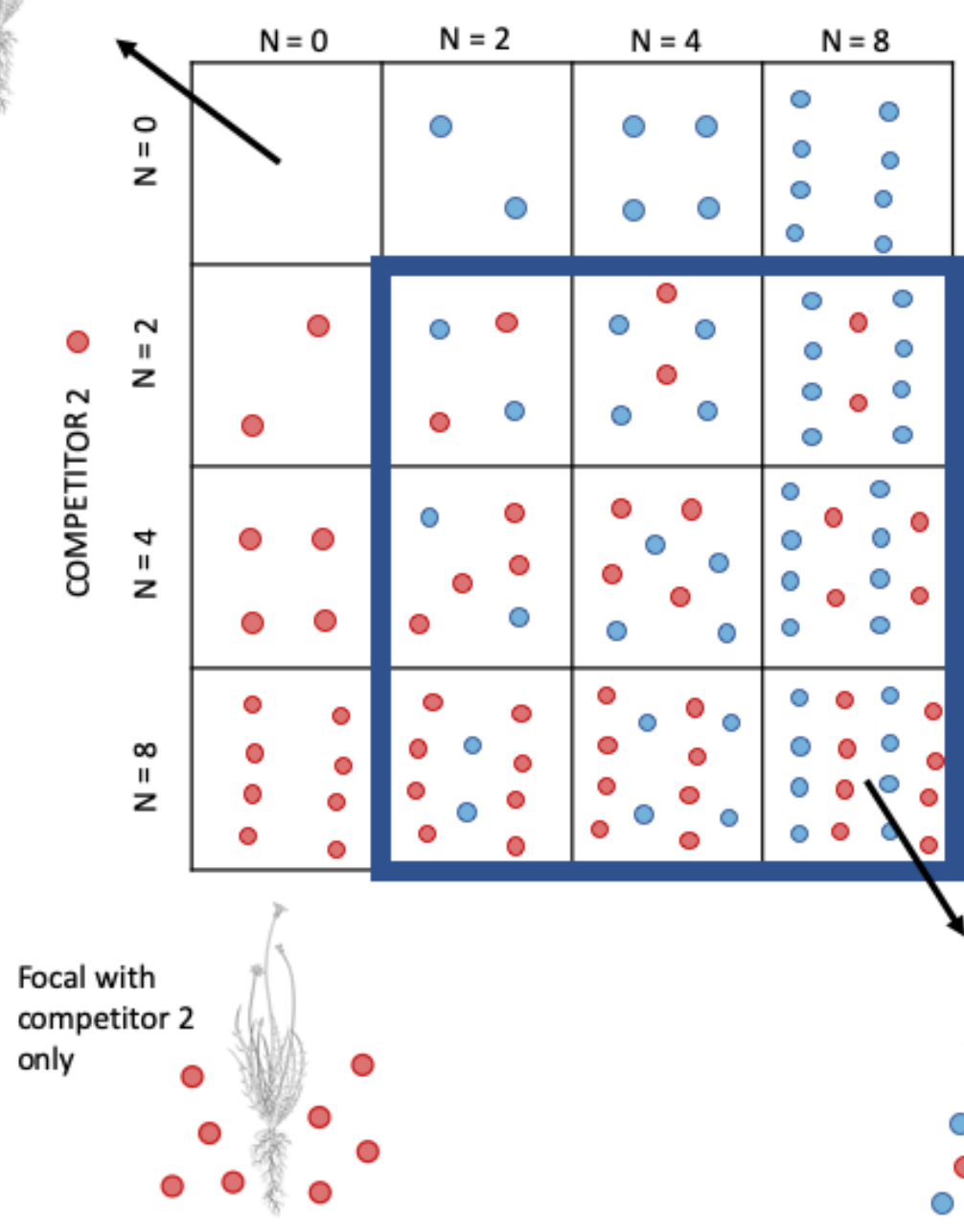

Focal with competitor 1 only
HOls possible between competitors 1 and 2
Focal with competitors 1 and 2

577 Figure 2 Schematic of orthogonal competition experiment required to detect higher

578 order interactions. Each square represents a separate study plot. Competitor 1, (blue

579 circles) and Competitor 2 (red circles) are grown in a gradient of increasing density

580 alone and together. A single individual of the focal species (line drawing) is grown in 
bioRxiv preprint doi: https://doi.org/10.1101/857920; this version posted November 29, 2019. The copyright holder for this preprint (which was not certified by peer review) is the author/funder, who has granted bioRxiv a license to display the preprint in perpetuity. It is made available under aCC-BY-NC-ND 4.0 International license.

581 each plot allowing the response to competition from each competitor species to be

582 fitted. 
B)
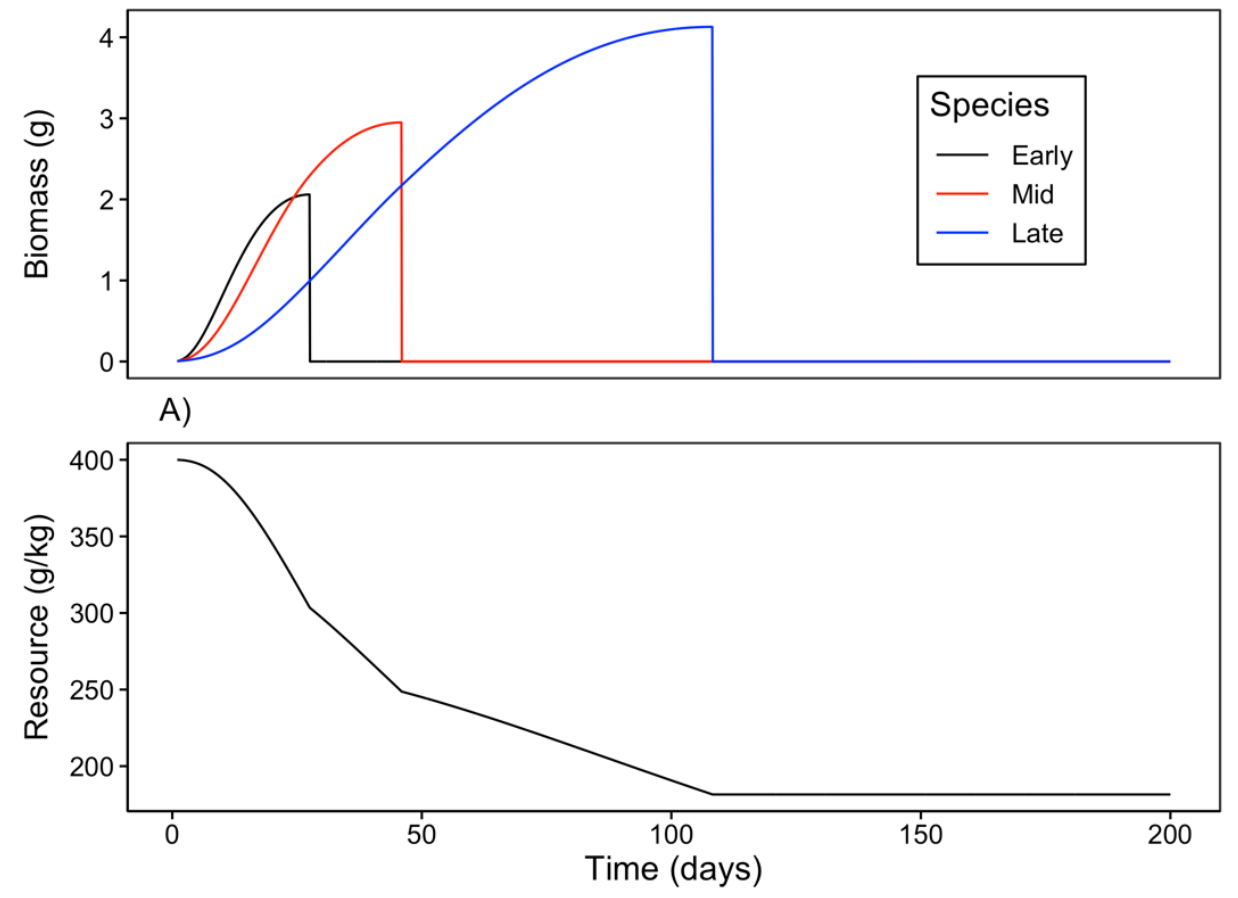

584 Figure 3. Example time course of A) annual plant growth and B) resource

585 concentration during a single simulated growing season. In this example, each

586 species' population consists of a single individual. The early season species (black)

587 grows rapidly when resource availability is high and senesces early. By contrast, the

588 late season species (blue) grows more slowly but grows later into the season as

589 resource availability declines. The growth curve for the mid-season species (red) lies

590 between these extremes. 


\section{Focal Species}

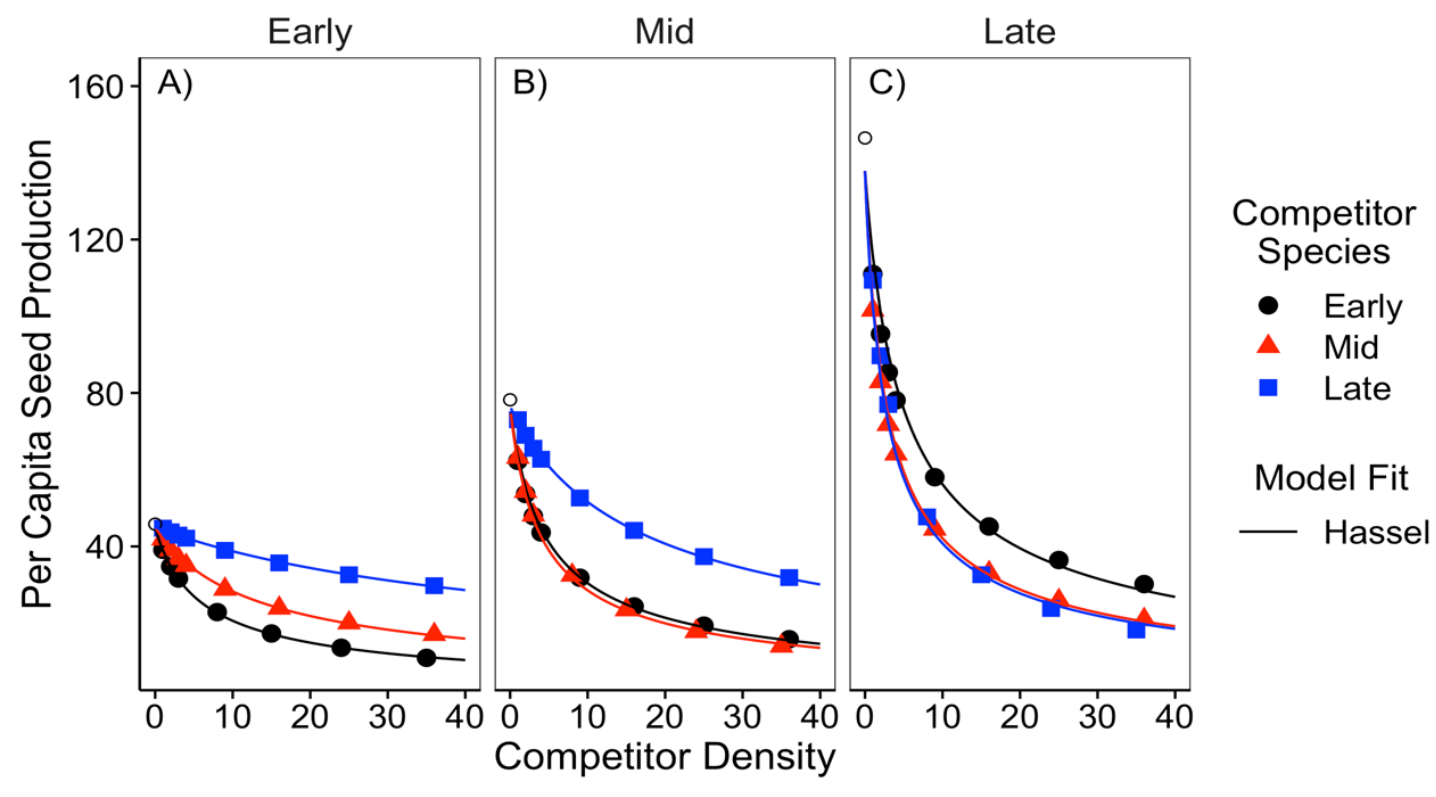

592 Figure 4. Simulated per capita seed production of the A) early, B) middle and C)

593 late season species in response to a single competitor species at a time. Competitor

594 density is shown on the $\mathbf{x}$-axis. Colors and shapes indicate the identity of the

595 competitor species. Open circles show the per capita seed production of each focal

596 species in the absence of any competitors. The solid line shows the fit of the Hassel

597 model. 


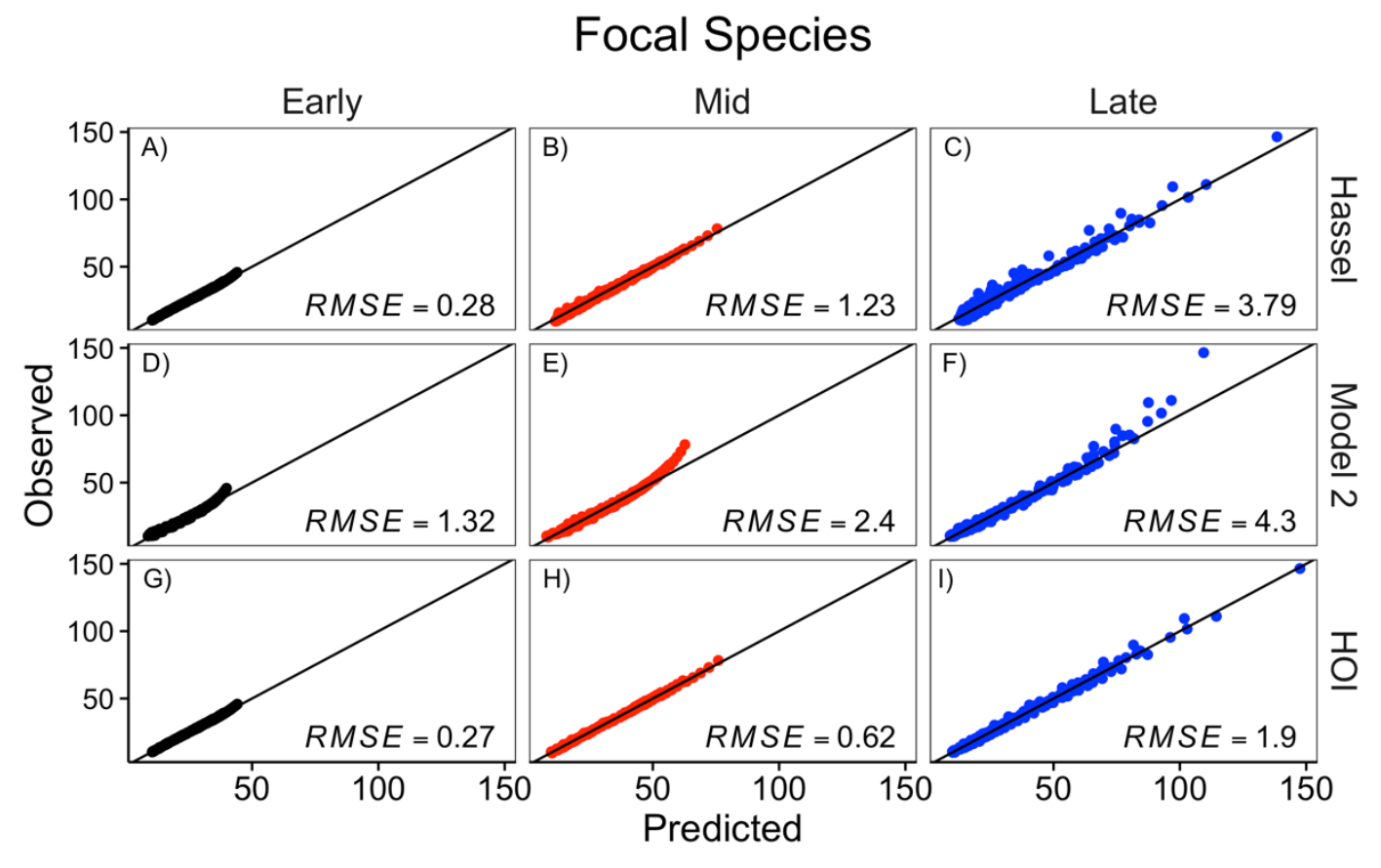

599 Figure 5. Comparison of the Hassel, multiplicative ('model 2'), and HOI models fit

600 to each focal species. The y-axis shows the simulated per capita seed production of

601 the focal species. The $x$-axis shows the per capita seed production predicted by the

602 phenomenological model. The top row, A-C, shows the prediction for the pairwise

603 Hassel model (eq. [5]); the middle row, D-F, shows the prediction from the

604 multiplicative model (eq. [11]); and the bottom row, G-I, shows the prediction from

605 the HOI model (eq. [10]). The one-to-one line and root-mean-squared error (RMSE)

606 for predictions from each model are shown. 


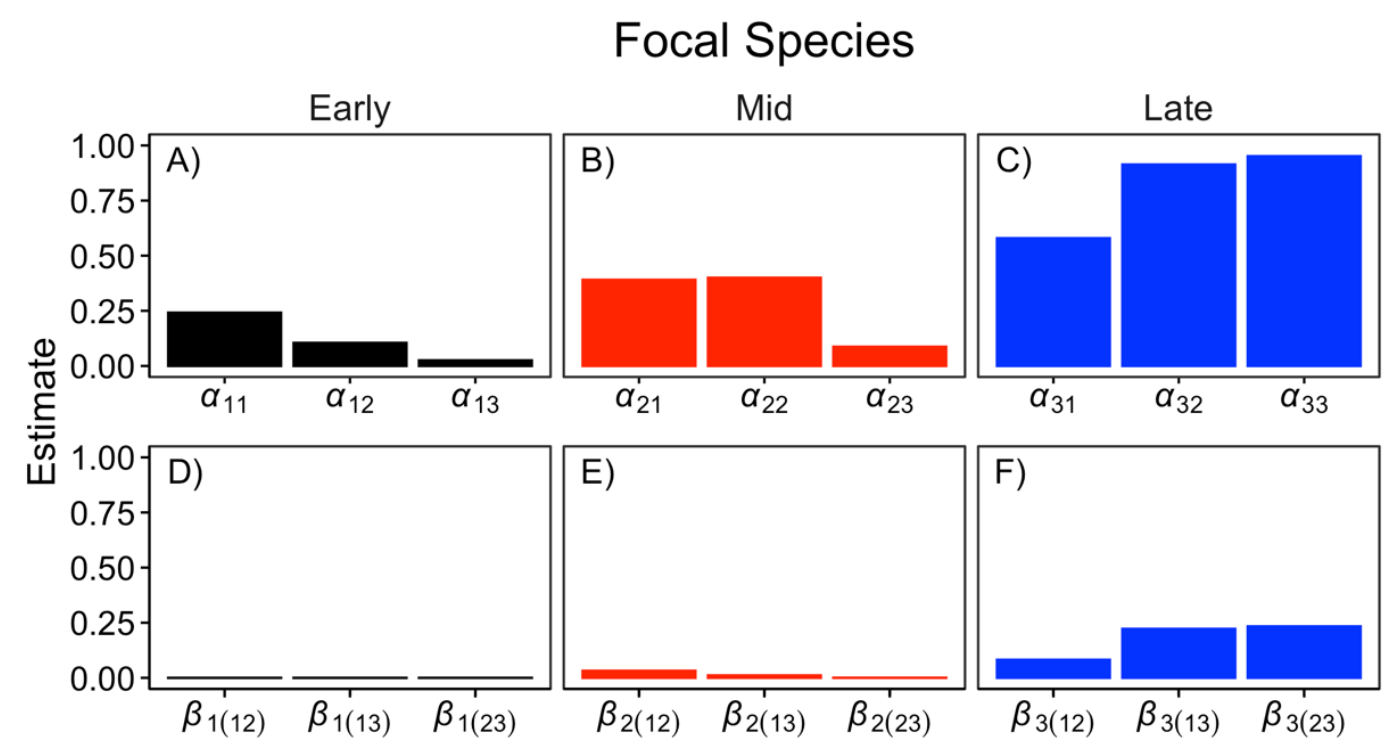

607

Parameter

608 Figure 6. Interaction coefficients for each of focal species from the HOI model. The

609 top row, A-C, shows the pairwise competition coefficients for the focal species and

610 each competitor. The bottom row, D-F, shows the two-species HOI coefficients.

611 Coefficient subscripts indicate which focal species and competitor species are

612 involved, $1=$ Early, 2 = Mid, $3=$ Late. 


\section{Supporting Information - Additional Tables}

\section{Table $\mathbf{S} 1$ Table of parameter values used in the growth simulation experiment in}

\section{5 the main text.}

\begin{tabular}{cll} 
Parameter & Value & Definition \\
\hline \hline$U$ & 200 & Duration of growth simulation (days) \\
$R(0)$ & 0 & Resource supply rate $\left(\mathrm{g} \mathrm{day}{ }^{-1}\right)$ \\
$d_{1}$ & 0.06 & Initial resource concentration $\left(\mathrm{g} \mathrm{kg}^{-1}\right)$ \\
$d_{2}$ & 0.12 & Mid competitor root density $\left(\mathrm{g} \mathrm{cm}^{-3}\right)$ \\
$d_{3}$ & 0.36 & Late competitor root density $\left(\mathrm{g} \mathrm{cm}^{-3}\right)$ \\
$\delta_{1}$ & 0.3 & Early competitor loss and respiration rate $\left(\mathrm{g} \mathrm{g}^{-1} \mathrm{~d}^{-1}\right)$ \\
$\delta_{2}$ & 0.15 & Mid competitor loss and respiration rate $\left(\mathrm{g} \mathrm{g}^{-1} \mathrm{~d}^{-1}\right)$ \\
$\delta_{3}$ & 0.053 & Late competitor loss and respiration rate $\left(\mathrm{g} \mathrm{g}^{-1} \mathrm{~d}^{-1}\right)$ \\
$K$ & 350 & Resource half-saturation constant $\left(\mathrm{g} \mathrm{kg}^{-1}\right)$ \\
$V_{\max }$ & 1 & Maximum resource conductance $\left(\mathrm{g} \mathrm{d}^{-1} \mathrm{~cm}^{-2}\right)$ \\
$p$ & 0.5 & Ratio of root biomass to total biomass \\
$v$ & 0.66 & Scaling exponent (unitless) \\
$q$ & 0.2 & Biomass assimilation rate $\left(\mathrm{g} \mathrm{g} \mathrm{g}^{-1}\right)$ \\
$\mu$ & 0.005 & Seed mass (g per seed) \\
$c$ & 0.1 & Conversion of final biomass to seed mass $\left(\mathrm{g} \mathrm{g}^{-1}\right)$ \\
\hline
\end{tabular}

616

617 
618 Supporting Information - Additional figures

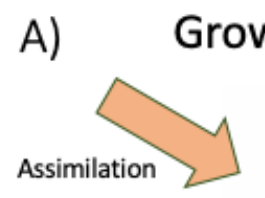

Growth Model

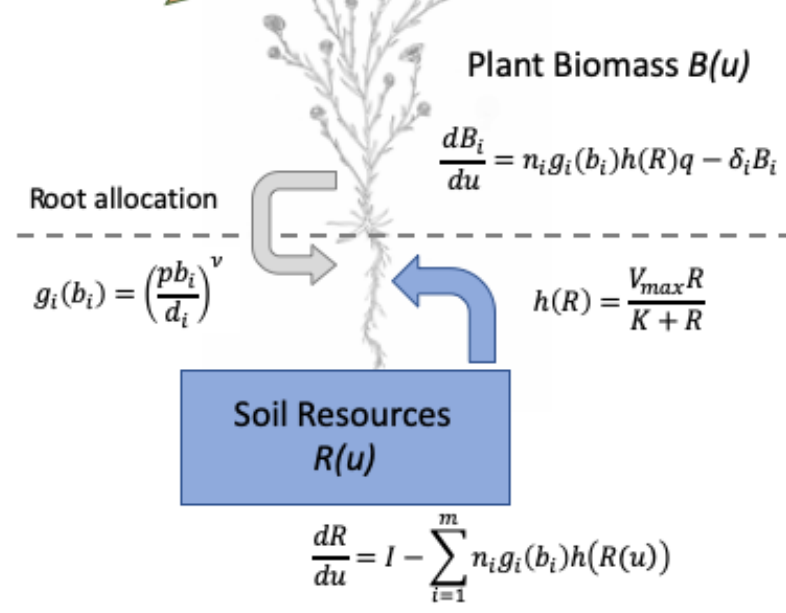

B) Reproduction

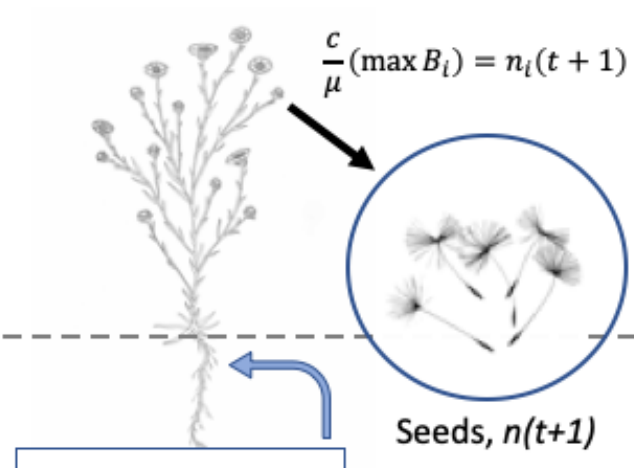

Soil Resources

$R(u)$

619

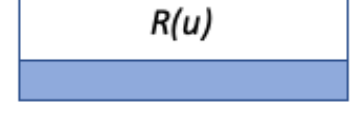

620 Figure S 1 Diagram schematic of annual plant growth model used in simulation. A)

621 in the model each individual plant start as a seed, grows over the course of a single

622 growing season. Growth is a function of plant biomass, root surface area and soil

623 resource availability. B) Over time the soil resources are depleted and plant growth

624 slows down. Plants reach a maximum size when losses due to respiration and tissue

625 senescence are greater than growth. At this point the plants convert stored

626 resources to seeds. The number of seeds in the next growing season is determined as

627 the total mass of seeds produced per species divided by the weight of a single seed. 
628 Supporting Information - Additional figures

\section{Focal Species}
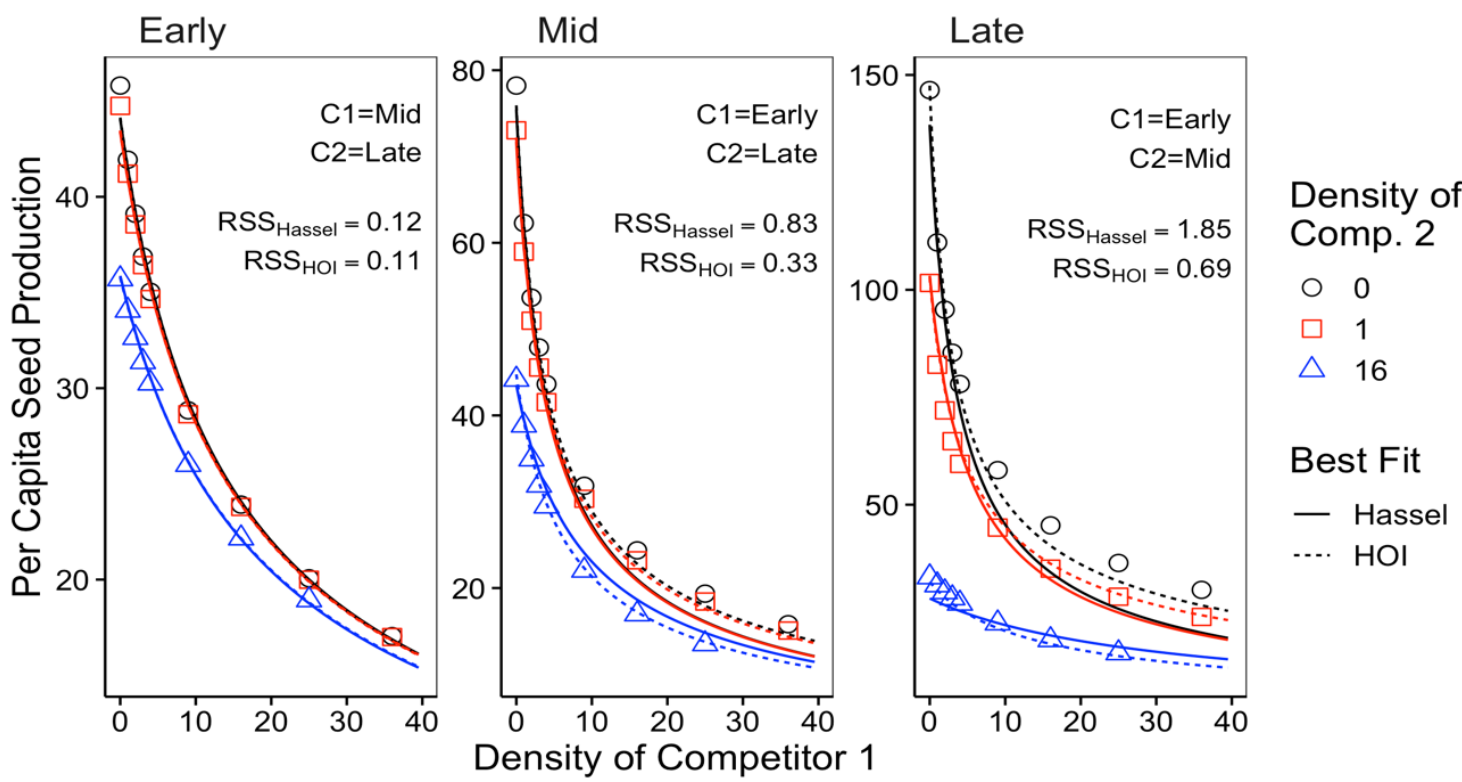

630 Figure S 2 Simulated per capita seed production of the A) early, B) mid and C) late

631 season species in response to density of two interspecific competitors. Densities of

632 two competitors are shown in each panel- the $x$-axis shows the density of the first

633 competitor, while different colored lines and shapes show the density of a second

634 competitor. Text in each panel lists the identities of competitor one and two (early,

635 mid or late). Lines show best fit from phenomenological models. Residual sum of

636 squared error is shown for each model and focal species. 


\section{Appendix A - The effect of trait differences on higher order interactions}

639 higher order interactions (HOIs) was associated with the magnitude of functional

640 differences between competitor species. We started with the same parameter values as in

641 the simulation in the main text in which there was a large difference between the species

642 in root density $\left(d_{i}\right)$ and tissue respiration rate $\left(\delta_{i}\right)$. In four additional simulation scenarios,

643 we gradually decreased the average difference between species in these traits (Table A1).

644 Specifically, we held the traits of the mid-season species constant and decreased the

645 difference in the root density trait, $d$, between the early and late-season species. We

646 assumed a trade-off between root density and tissue respiration rate such that changing

647 root density was accompanied by a change in tissue respiration rate, $\delta_{i}$ (Figure A1). We

648 quantified the average functional difference between species as the standard deviation of

649 root density among all species. In each scenario, we simulated competition and fitted the

650 phenomenological HOI model as in the main text. For each species in each scenario, we

651 quantified the strength of HOIs as the average magnitude of the $\beta$ coefficients divided by

652 the average magnitude of the $\alpha$ coefficients. For the mid and late season species, the

653 strength of the HOIs increased with the functional difference between species (Figure A1

$654 \mathrm{~B} \& \mathrm{C}$ ). For the early season species, HOIs were weak in all five scenarios (Figure A1 A).

655 These simulations show that the functional differences between competitors drive the

656 HOIs we observed in this system. 
657 Table A 1. Parameter values for five simulations with gradually decreasing the trait

658 difference between the early season and late season species. All other simulation

659 parameters are the same as in Table S1.

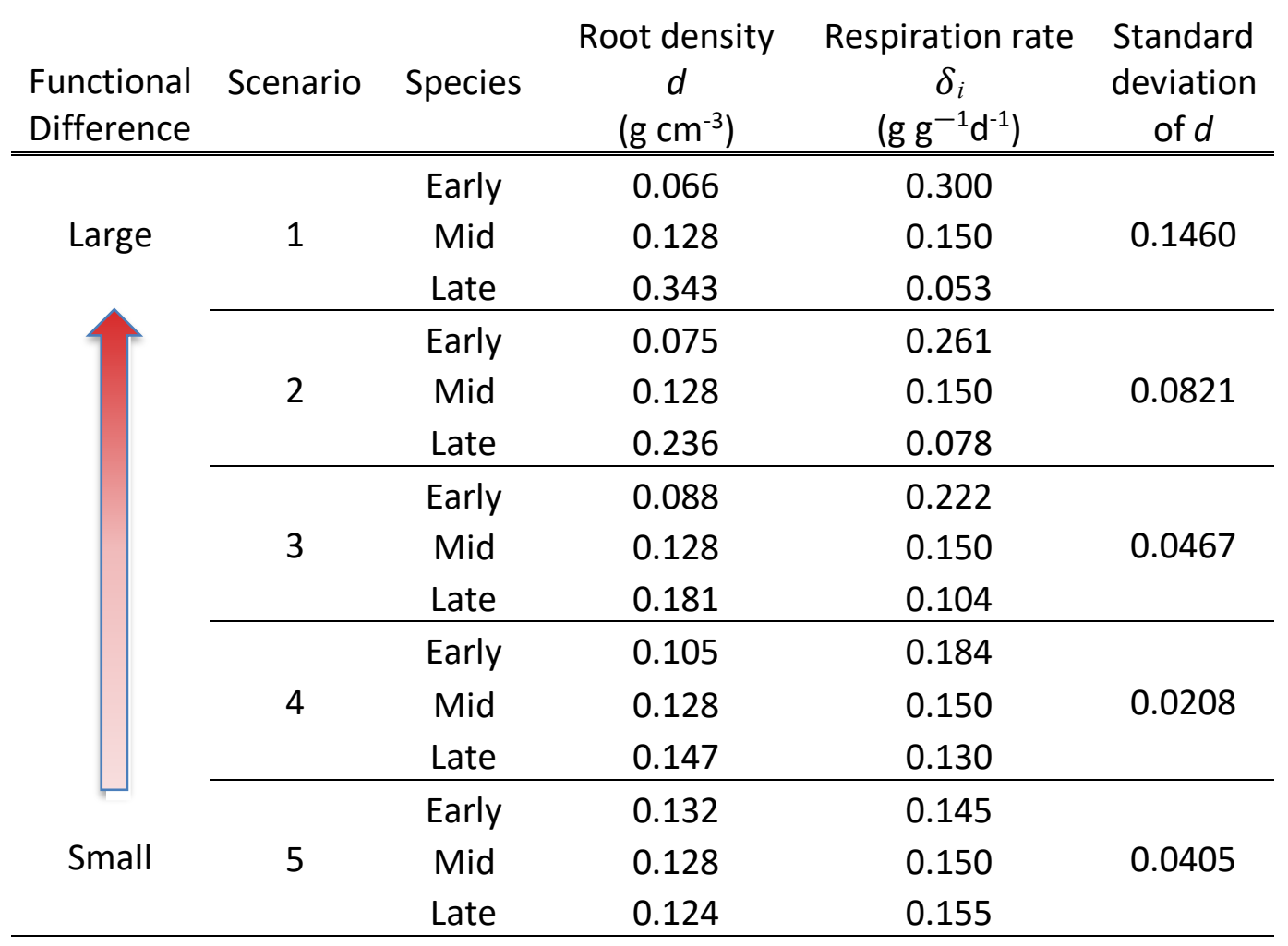

660 

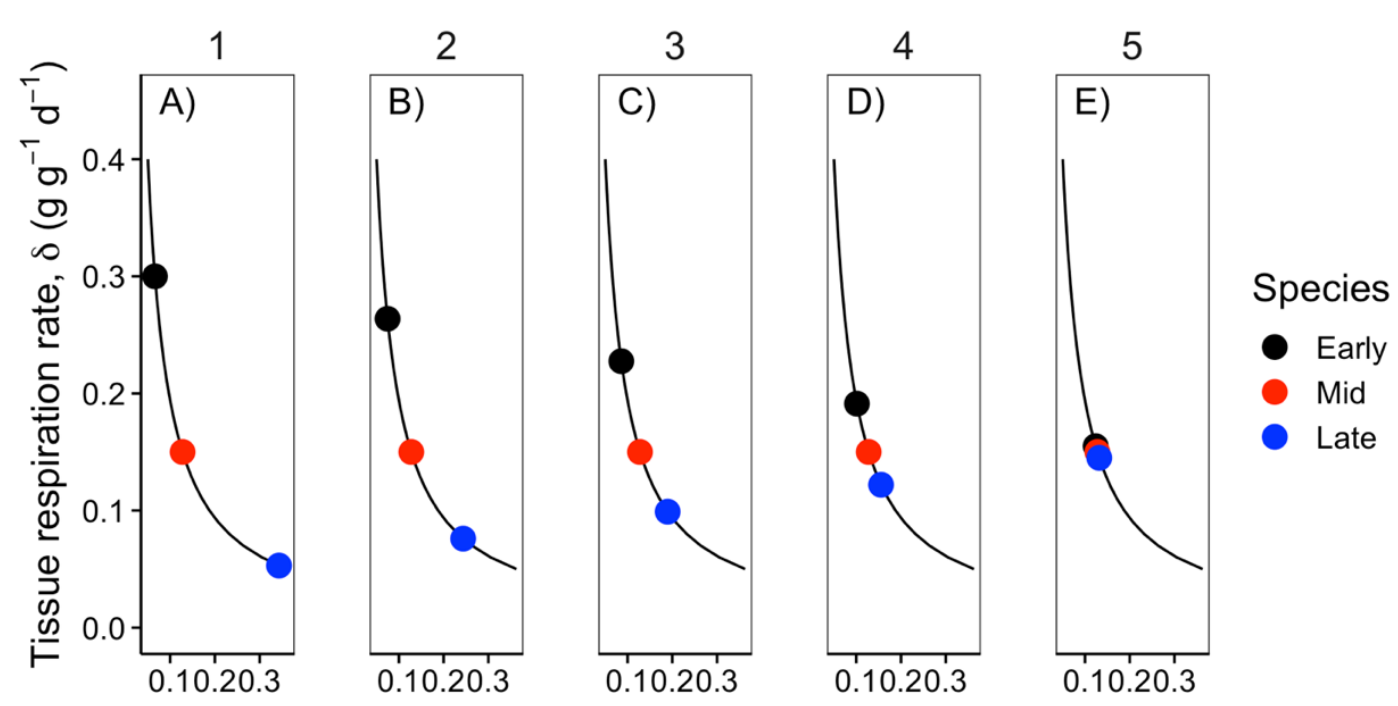

Root tissue density, $d\left(\mathrm{~g} \mathrm{~cm}^{-3}\right)$

661

662 Figure A 1. Colored points show the value of functional traits, root density and

663 tissue loss rate, for each of the three species in each of the five simulation scenarios

664 (A-E). Across the five scenarios, the differences between the early season and late

665 season species' root density and respiration rates were gradually decreased. The

666 mid-season species' traits were held constant. The black line indicates the trade-off

667 between the root density and tissue respiration rate traits. 

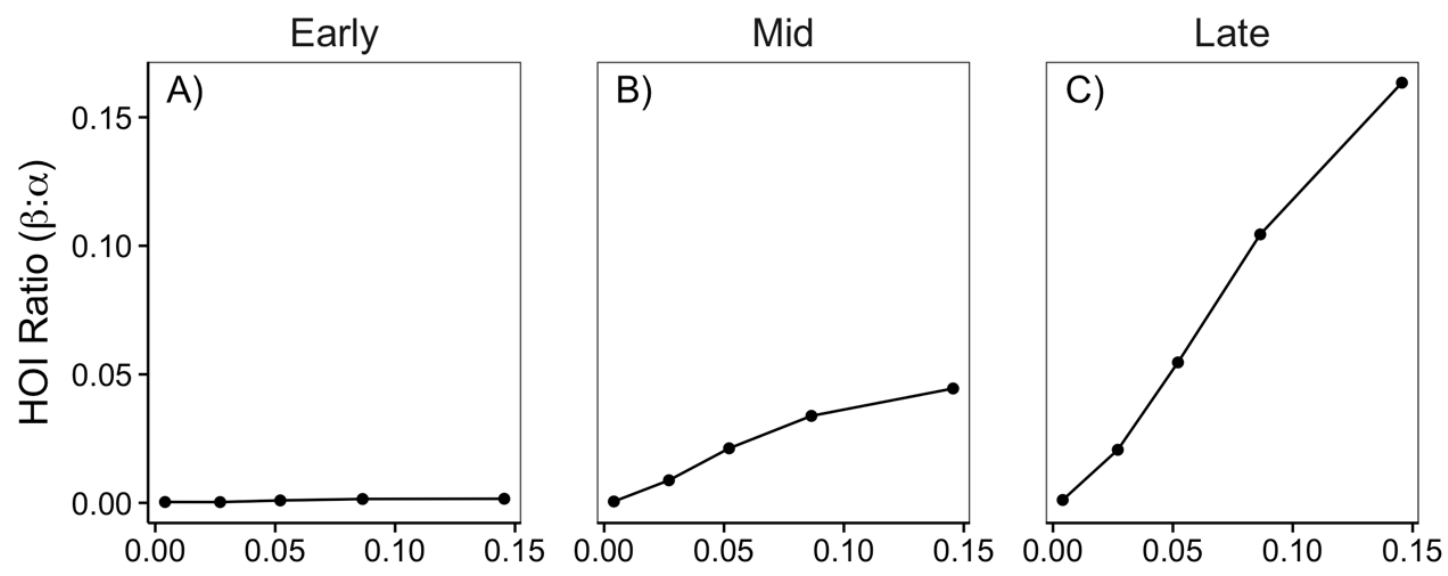

Community Trait Difference (std. dev. $d$ )

669 Figure A 2. The strength of HOIs depends on the difference in species functional

670 traits. The y-axis quantifies the strength of HOIs affecting the early (A), mid (B) and

671 late (C) species as the ratio of the of the average magnitude of the $\boldsymbol{\beta}_{i(j k)}$ coefficients to

672 the average magnitude of the $\alpha$ coefficients in the phenomenological HOI model. A

673 larger ratio $\beta$ : $\alpha$ ratio indicates stronger HOIs compared to pairwise interactions.

674 The $\mathbf{x}$-axis quantifies the community-level trait difference as the standard deviation

675 of the root density trait, $d$.

676 